\title{
A method for detection of structure ${ }^{\star}$
}

\author{
M. Gustafsson ${ }^{1}$, J. L. Lemaire ${ }^{2}$, and D. Field ${ }^{1}$ \\ 1 Department of Physics and Astronomy, University of Aarhus, 8000 Aarhus C, Denmark \\ e-mail: maikeng@phys.au.dk \\ 2 Observatoire de Paris \& Université de Cergy-Pontoise, LERMA \& UMR 8112 du CNRS, 92195 Meudon, France
}

Received 9 March 2006 / Accepted 23 May 2006

\begin{abstract}
Context. In order to understand the evolution of molecular clouds it is important to identify the departures from self-similarity associated with the scales of self-gravity and the driving of turbulence.

Aims. A method is described based on structure functions for determining whether a region of gas, such as a molecular cloud, is fractal or contains structure with characteristic scale sizes.

Methods. Using artificial data containing structure it is shown that derivatives of higher order structure functions provide a powerful way to detect the presence of characteristic scales should any be present and to estimate the size of such structures. The method is applied to observations of hot $\mathrm{H}_{2}$ in the Kleinman-Low nebula, north of the Trapezium stars in the Orion Molecular Cloud, including both brightness and velocity data. The method is compared with other techniques such as Fourier transform and histogram techniques. Results. It is found that the density structure, represented by $\mathrm{H}_{2}$ emission brightness in the $K$-band $(2-2.5 \mu \mathrm{m})$, exhibits mean characteristic sizes of 110, 550, 1700 and 2700 AU. The velocity data show the presence of structure at 140,1500 and 3500 AU. Compared with other techniques such as Fourier transform or histogram, the method appears both more sensitive to characteristic scales and easier to interpret.
\end{abstract}

Key words. ISM: individual objects: OMC1 - ISM: structure - ISM: kinematics and dynamics

\section{Introduction}

The velocity and density fields of molecular clouds have been observed to show very complex structure. This property suggests that structure is dominated by turbulence and this has been verified by both observations and numerical simulations (e.g. Falgarone \& Phillips 1990; Boldyrev et al. 2002a); see also the extensive reviews by Elmegreen \& Scalo (2004) and Mac Low \& Klessen (2004). Clouds dominated by turbulence lack any characteristic scale and are inherently self-similar with a variety of fractal dimensions. This has been observed over a broad range of scales, 0.02-100 pc (e.g. Larson 1981; Chappell \& Scalo 2001; Ossenkopf \& Mac Low 2002).

Self-similarity and lack of characteristic scale in molecular clouds must however break down at some dimension or range of dimensions associated with star formation, as demonstrated in Vannier et al. (2001) and Gustafsson et al. (2006). Turbulence creates local clumps of gas and this process, known as turbulent fragmentation, is considered the root cause of observed density structure and may give rise to large density contrasts. Star formation begins to take place at smaller scales in gravitationally unstable clumps. The distribution of sizes of these clumps is directly related to the initial mass function (IMF) for star formation (Padoan \& Nordlund 2002). The predominantly turbulent era, characterized by lack of preferred scale, is expected to end when gravitation becomes dominant in clumps. At this stage,

^ Partly based on observations obtained at the Canada-France-Hawaii Telescope (CFHT) which is operated by the National Research Council of Canada, the Institut National des Science de l'Univers of the Centre National de la Recherche Scientifique of France, and the University of Hawaii and on observations obtained at the ESO/VLT, Proposal 70.C-0315. the density and velocity fields are not fractal, but will contain characteristic scales. Hence certain scales may be overpopulated or under-populated relative to the model of a simple Kolmogorov-type cascade (Kolmogorov 1941; Frisch 1995). The range of scales, at which clumps become gravitationally bound and turbulence looses its kinematically dominating effect, is important for both theories of star formation and of turbulence.

Characteristic scales may also be imposed on the medium by other mechanisms than gravitational collapse. In particular energy may be injected into the medium at the largest scales by supernova explosions or at the tens of thousands of AU scale by outflows from massive stars or from low mass stars at scales of a few thousand AU. In order to understand the evolution of molecular clouds it is essential to identify the presence of these energy injection processes and the scales at which they occur.

The purpose of the present paper is to introduce a technique, based on structure functions (SFs), to identify characteristic scales in the interstellar medium - or indeed in any medium. The technique appears more sensitive to characteristic scales, for example in spatial brightness or velocity, than methods presently used. The method is relatively easy to interpret in terms of preferred scale sizes and is as easy to implement as existing methods.

Experience has proven that it is non-trivial to establish the scale(s) at which self-similarity begins to break down and an array of techniques as broad as possible should be made available. For example, four different methods were used in Vannier et al. (2001) in order to demonstrate that self-similarity breaks down at star-forming scales of around 500-1000 AU in a small portion of the Orion Molecular Cloud (OMC1). The present contribution should be seen in this light, not as a panacea for structure identification but rather as another tool for this purpose. 
The methods currently available are those of (i) Blitz \& Williams (1997), who introduced a method of degrading the spatial resolution and establishing the presence of preferred scales from histograms of the degraded images, a method used for example in Vannier et al. (2001) and Lacombe et al. (2004), (ii) Fourier transform power spectra, used to pick out frequencies and hence scales in images (e.g. Vannier et al. 2001), (iii) clump decomposition (Stutzki \& Guesten 1990), (iv) $\Delta$-variance analysis (Stutzki et al. 1998; Bensch et al. 2001), (v) wavelet transformation (e.g. Farge 1992).

The non-fractal nature of molecular clouds at the starforming scale of 500-1000 AU was first detected in Vannier et al. (2001) for Orion. Essentially the same preferred scales between 500 and $1100 \mathrm{AU}$ are also reported in Lacombe et al. (2004). Non-fractal nature has also been reported at larger scales in Blitz \& Williams (1997) who found a preferred scale of $0.25-0.5 \mathrm{pc}\left(5-10 \times 10^{4} \mathrm{AU}\right)$ in Taurus. Material may however be self-similar down to a few $\times 10^{-4} \mathrm{pc}(<100 \mathrm{AU})$ in diffuse material, with clumps of mass $\sim 10^{-7} M_{\odot}$, as found in the photodissociation region around the B-star HD 37903 in NGC 2023 (Rouan et al. 1997). Here we show that structure functions (SFs) or local derivatives of SFs provide a powerful way to detect preferred scales in a medium. The method is tested on observations of $\mathrm{H}_{2}$ emission in $\mathrm{OMC} 1$.

\section{Method}

The SFs of order $p$ of a spatially resolved parameter $A$ are defined as

$S_{p}(r)=\left\langle\left|A\left(r^{\prime}\right)-A\left(r^{\prime}-\delta\right)\right|^{p}\right\rangle=\left\langle|\Delta A|^{p}\right\rangle$

where the average is taken over all map positions $r^{\prime}$ and all distances $\delta$ such that $|\delta|=r$. The parameter $A$ can for example be velocity or brightness. The SFs are measures of the spatial correlations of the parameter $A$. A completely random distribution would result in a constant structure function as a function of $r$, whereas for example a gradient in the image would lead to a structure function increasing with $r$.

Structure functions have traditionally been used in turbulence theory to quantify the properties of the turbulence. In a predominantly turbulent medium where the velocity and density fields are self-similar or fractal, the SFs are well described by power laws, $S_{p}(r) \propto r^{\zeta(p)}$ in the inertial range between the driving and dissipation scale. This is found from both theory (Kolmogorov 1941; Boldyrev et al. 2002b) and simulations (e.g. Boldyrev et al. 2002a; Kritsuk \& Norman 2004) and simply conveys that in turbulent regions, the velocity field, say, is spatially correlated in such a way that larger separations correspond to larger velocity differences on average. This is in fact a manifestation of the turbulent energy cascade originally proposed by Kolmogorov.

On the other hand, if the region in question is not self-similar, but contains structure with a preferred scale, the SFs will deviate from power-laws. It is shown here that these deviations are a sensitive test of the presence of structure and may be used to reveal the size of structures. If the region contains only a single structure in the map of parameter $A$ of a certain size, $R$, it is evident that correlations will only persist up to scale $R$. In the structure function this will show up as an increase of $S_{p}(r)$ at scales $<R$ and a constant value at scales $>R$. The same behaviour will be found if there is a large number of structures with the same size randomly distributed in the map. When clumps with two or more distinct preferred sizes are present, which is most likely the case in star forming regions, the structure function will show this effect for all sizes involved. The effect on the SFs will become successively less pronounced at any specific size as more scales are identified. In a real system, structures will most likely tend to group around a preferred scale with some characteristic deviation from a mean value. This aspect turns out to be more of a gloss on the present description than a fundamental point but can lead to systematic errors in structure size determination. This is described in Sect. 4.4.

To test the ideas set out above, three maps have been constructed with i) a single circular clump with a diameter of 40 pixels and an intensity distribution given by the paraboloid function

$f(x, y)=C\left(1-\frac{\left(x-x_{0}\right)^{2}+\left(y-y_{0}\right)^{2}}{r^{2}}\right)$

where $r$ is the radius of the clump, $\left(x_{0}, y_{0}\right)$ is the centre position and $C$ is the peak intensity; ii) 100 randomly distributed clumps with the same characteristics as in i) and iii) 100 clumps with a diameter of 20 pixels and 20 clumps with a diameter of 100 pixels where again all clumps were randomly distributed and of paraboloid shape. In all cases the clumps have been distributed on a square 1024 by 1024 grid. Similar maps were also constructed using conic shapes $\left(f(x, y)=C\left(1-\frac{\sqrt{\left(x-x_{0}\right)^{2}+\left(y-y_{0}\right)^{2}}}{r}\right)\right)$ for the intensity distribution in the clumps.

The SFs of order 1-5 for case (i) are shown in Fig. 1a. The SFs become roughly constant at $r \geq 30$ pixels close to the expected value of 40 . The SFs for case (ii) are essentially the same. They are not shown here. The effects on the SFs caused by the presence of clumps are more clearly seen at higher orders.

Preferred scale sizes in a map or an image result in changes in the logarithmic slope of SFs. Therefore the local logarithmic derivatives, $\operatorname{dlog} S_{p}(r) / \operatorname{dlog} r$, highlight the deviations from fractality and thus we propose to use these derivatives to detect such scales. As seen above the presence of preferred scales causes a decrease in the slope of the SFs making it roughly constant near the relevant size. This results in a plateau in the logarithmic derivatives if larger scales are not present, or a minimum if such scales are present. The former case is demonstrated in Fig. 1b for case (i) above, that is, for a single clump, and in Fig. 1c for case (ii), that is, for 100 such clumps. A minimum is seen at $\sim 35$ pixels in both Figs. $1 \mathrm{~b}$ and $\mathrm{c}$, close to the imposed structure size of 40 pixels (see above). The inset to Fig. 1b displays the derivatives around $r \sim 35$ in detail and shows that the position of the minimum moves towards a lower value of $r$ when $\mathrm{p}$ is increased. This occurs because higher orders of the structure function give more weight to higher values of $\Delta A$ (see Eq. (1)), thus depressing the outer regions of clumps, which is the location of low values of $\Delta A$ in these simulations. Thus estimates of scale sizes from high order SFs represent lower bounds to the scale sizes and the estimated values can be $10-20 \%$ too low. In Fig. 1c, in addition to the first plateau or weak minimum at $\sim 35$ pixels, there is a secondary plateau at $\sim 75$ pixels, indicating the presence of a scale size approximately twice as large as the clumps in the simulation. This is most likely due to overlapping of the randomly placed individual clumps. By implication this method cannot distinguish between one large clump or several smaller clumps nearly coinciding in the same line-of-sight.

The local logarithmic derivatives for $p=1-5$ are shown in Fig. 2 for case (iii) involving 100 clumps of diameter 20 pixels and 20 clumps of diameter 100 pixels. Here a local minimum is seen at $\sim 17$ pixels and $\sim 100$ pixels, representing the scales of 20 and 100 pixels included in this test image. Note that two scale sizes will only show clearly if the scales are well separated. 

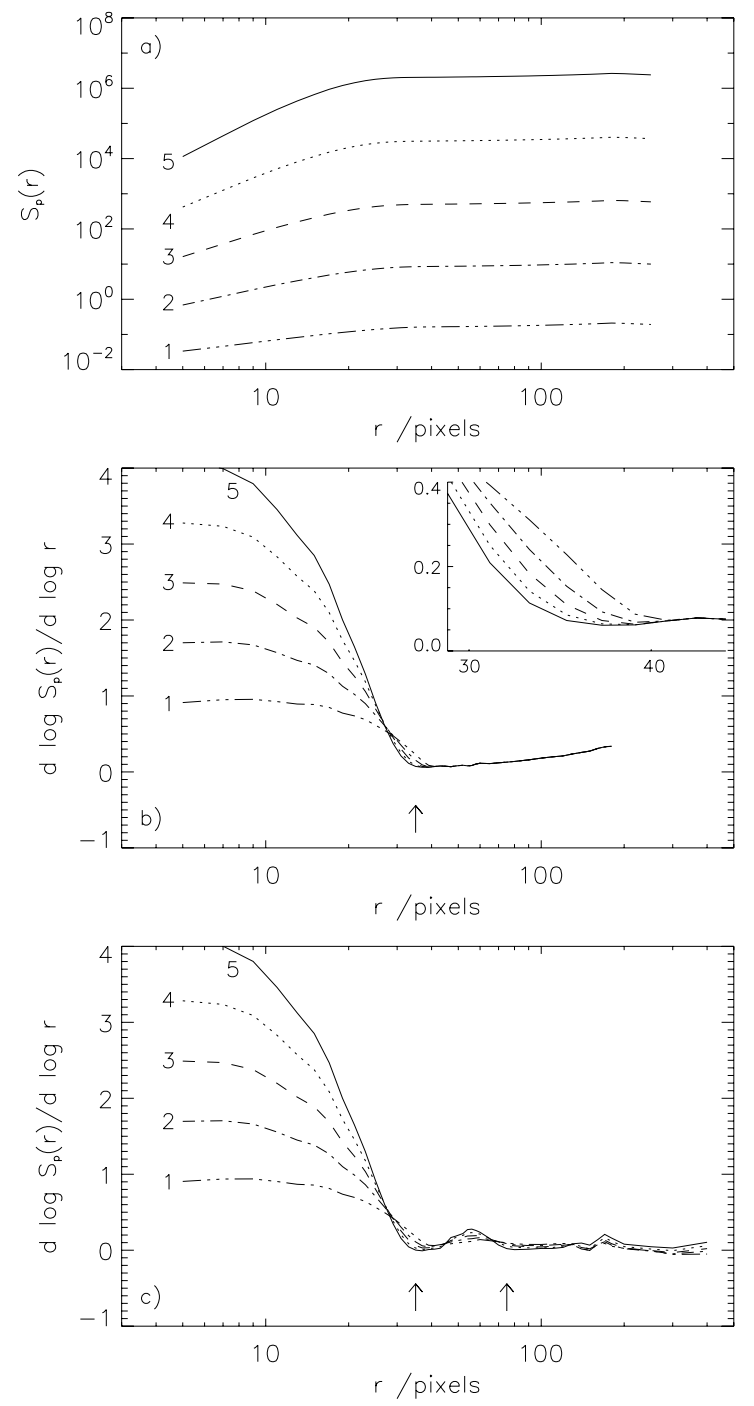

Fig. 1. a) Structure functions of order 1-5 (labelled accordingly) for a simulated map, case (i) (see text). b) Local logarithmic derivatives of the SFs shown in a) Inset: Blow-up of $r=29-45$ pixels. c) Local logarithmic derivatives of SFs of order 1-5 derived from the simulated map of case (ii).

If they are too close the larger scale will dominate and suppress the smaller. In real data this means that a detected scale size might conceal other smaller scales (see also Sect. 4.4). The inset to Fig. 2 shows the corresponding structure function for 5 th order, showing plateaux at $\sim 15$ pixels and at $>\sim 80$ pixels.

The same tests were performed on the maps of clumps with conical shaped intensity distributions and yielded similar results to those above. This suggests that effects seen in the structure functions are independent of the shape of the clumps. This is of course only valid for clump geometries which can at least crudely be characterized by a single dimension. Tests were also carried out on clumps with an ellipsoid shape with randomly oriented axes. These are characterized by two sizes, that is, those of the major and minor axes and the results are very similar to those of clumps with two different sets of diameters, that is case (iii) above.

An additional property is that in the presence of real structure the derivatives of structure functions of all orders tend to congregate around the same value at a local minimum or plateau. This provides a further characteristic which aids the detection

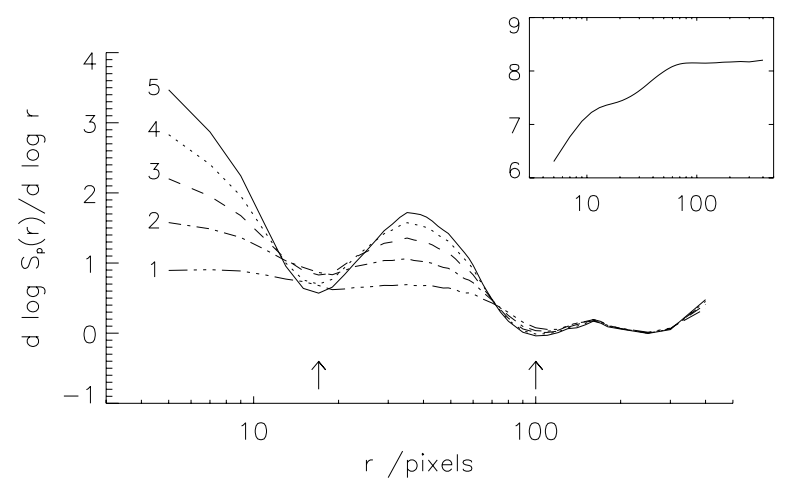

Fig. 2. Local logarithmic derivatives of SFs of order 1-5 from the simulated map of case (iii) (see text). Inset: the logarithm of the 5th order structure function of case (iii).

of structure. In numerical simulations of fully developed turbulence, Biferale et al. (2004) noted a similar tendency for the local derivatives to accumulate at a certain value.

A further point in assigning the presence of structure is the question of how large a structure may be detected for a given size of image, so called "edge-effects". In case (iii), in Fig. 2, there is already evidence for apparent structure at $\sim 250$ pixels which is not in the data. Edge-effects have been studied here using fractional Brownian motion structures (fBm-fractals). We have calculated SFs and logarithmic derivatives for a number of such fractal images. For these, the SFs are expected to be pure power laws and the derivatives should be independent of $r$. The value of the logarithmic derivative depends on the choice of power spectral index and the order of the structure function involved, being proportional to the latter. In passing we note that fractal systems cannot therefore yield logarithmic derivatives of SFs which congregate at a particular value.

An example of these test calculations is seen in Fig. 3 where SFs of order 1-5 and logarithmic derivatives for a fractal image of size $2048 \times 2048$ pixels are shown. The SFs may be approximated by power laws save at the largest scales. The logarithmic derivatives are nearly constant up to $r \sim 700$ pixels, any deviations from constancy below this value being due to the imperfect fractal nature of the simulations arising from pixelation. At around 700 pixels, a local minimum is found and the derivatives for $p=3-5$ congregate around the value of 0.4 , apparently indicating a preferred structure size. The same analysis has been carried out on fractal images of size $1024 \times 1024,512 \times 512$ and $256 \times 256$ pixels. In all images apparent structure at scales of $\sim 1 / 5-1 / 3$ of the size of the map was found. Hence increasing the size of the map also increased the absolute value of the apparent structure size. Thus we conclude that the apparent structure in the fractal images is an edge-effect and that detection of scale sizes much greater than $1 / 5$ of the size of the map should not be attempted as such scales cannot be reliably identified.

\section{Real data for test purposes}

In order to illustrate the method of structure analysis described here with real data, we use results for the emission from vibrationally excited $\mathrm{H}_{2}$ in $\mathrm{OMC} 1$, where excitation occurs largely through shock excitation (with a small photon excitation component: see e.g. Kristensen et al. 2003). The relevant data are reported in Gustafsson et al. (2003), Lacombe et al. (2004), Nissen et al. (2005) and Kristensen et al. (2006). These three datasets refer only to the hot component of the gas, above $\sim 1000 \mathrm{~K}$, but nevertheless form valuable datasets for the identification of 

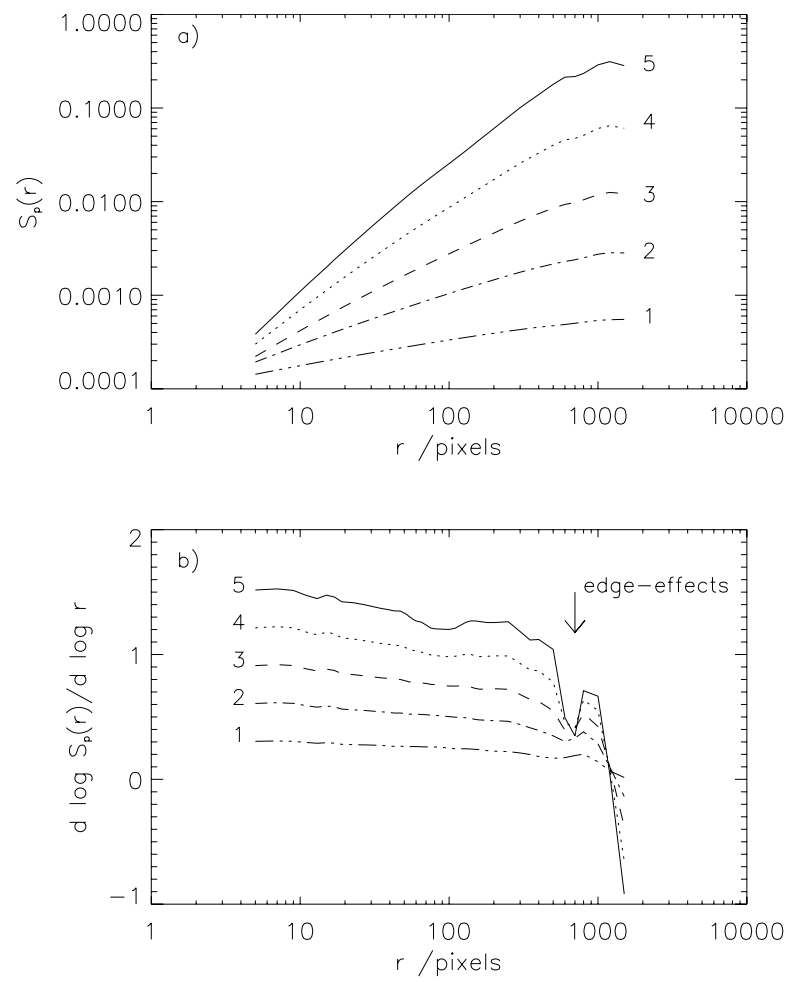

Fig. 3. a) Structure functions of order 1-5 (labelled) of fractal image. b) Corresponding local logarithmic derivatives of structure functions of order 1-5.

structure since they probe very small scales, from as low as $55 \mathrm{AU}$ (Lacombe et al. 2004), and also involve the major constituent of the gas, albeit in an excited state.

Data in Gustafsson et al. (2003) were obtained at the CFHT with a Fabry-Perot interferometer in conjunction with adaptive optics (GriF, Clénet et al. 2002) and contain both brightness and velocity information. Observations were performed in the NIR $K$-band by scanning the $v=1-0 S(1) \mathrm{H}_{2}$ emission line at $2.121 \mu \mathrm{m}$. The field of view was $36^{\prime \prime} \times 36^{\prime \prime}$ and the pixel scale is $00^{\prime} 035(1024 \times 1024$ pixel detector). The dataset consists of four spatial and velocity resolved images, which are amalgamated into one field of $89^{\prime \prime} \times 67^{\prime \prime}$ or $0.2 \times 0.15 \mathrm{pc}$ $\left(4.1 \times 10^{4} \times 3.1 \times 10^{4} \mathrm{AU}\right)$ given a distance to Orion of $460 \mathrm{pc}$ (Bally et al. 2000). The field is centred approximately on the Becklin-Neugebauer (BN) object $\left(05^{\mathrm{h}} 35^{\mathrm{m}} 14^{\mathrm{s}} 1,-05^{\circ} 22^{\prime} 22^{\prime \prime} \cdot 9\right)$. The spatial resolution is 0 ' $^{\prime} 15(70 \mathrm{AU})$ and relative velocities between adjacent resolution elements are determined to between $0.3 \mathrm{~km} \mathrm{~s}^{-1}(1 \sigma)$ in bright regions and $8.4 \mathrm{~km} \mathrm{~s}^{-1}$ in the weakest regions considered here. A detailed description of data acquisition and reduction may be found in Gustafsson et al. (2003) with further discussion in Gustafsson et al. (2006). We will refer to this dataset as the CFHT/GriF data.

Data in Kristensen et al. (2006) were also obtained at the CFHT, with the PUEO adaptive optics and the KIR infrared detector but without velocity information and covering a more restricted region than the CFHT/GriF data. Emission from the $\mathrm{H}_{2}$ $1-0 S(1)$ line was isolated using a narrow-band filter $(\lambda / 100)$. Continuum was recorded with a filter centred at $2.183 \mu \mathrm{m}$ and subtracted from the data obtained in the $S(1)$ filter, data being subjected to standard reduction procedures (Kristensen et al. 2006). The dataset used here is that designated as $\mathrm{W}$ in Kristensen et al. (2006) centered $\sim 18^{\prime \prime}$ south-west of BN. The field is $36^{\prime \prime} \times 36^{\prime \prime}$, the pixel scale is $0{ }^{\prime} 035$ and the resolution
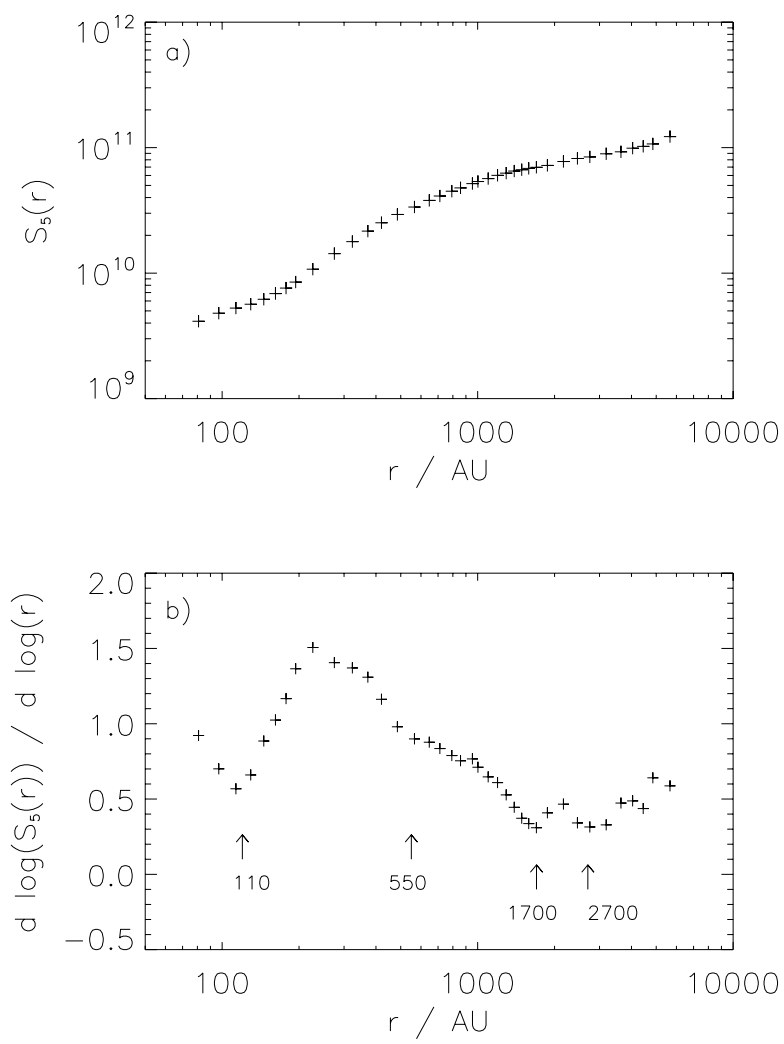

Fig. 4. a) The 5th order structure function of velocity integrated brightness in OMC1 from CFHT/GriF data. b) Corresponding local logarithmic derivatives of the structure function of order 5 .

is 0'.45 (200 AU). This dataset will be referred to as CFHT/PUEO-KIR.

Data in Lacombe et al. (2004) were obtained with VLT/NACO. The $\mathrm{H}_{2} 1-0 S(1)$ line was isolated using a narrowband filter, with continuum subtraction as described in Lacombe et al. (2004). The observations used here are those designated as the east-south-east (ESE) field, $27^{\prime \prime} \times 27^{\prime \prime}$ with a pixel scale of $0{ }^{\prime} 027$ and a resolution of $0 !^{\prime} 12(55 \mathrm{AU})$. This corresponds to the field analysed in Vannier et al. (2001) and is referred to here as the VLT/NACO data. This field lies to the east of the CFHT/PUEO-KIR field.

In the succeeding sections we use the new technique employing SFs for the various datasets as follows. First we use brightness data extracted from the CFHT/GriF. These data cover the greatest physical extent of any of our datasets. At this stage we ignore the velocity information in these data. We then consider the CFHT/PUEO-KIR and VLT/NACO data. In the latter two cases we compare our results with those of the histogram method of Blitz \& Williams (1997) and the Fourier transform method. In a third section we use the velocity data contained in the CFHT/GriF results.

\section{Identification of structure in OMC1}

\subsection{Use of the method with CFHT/GriF $\mathrm{H}_{2}$ brightness data}

We use the CFHT/GriF observations described in Sect. 3 to show that the molecular gas in OMC1 contains a number of preferred scales, illustrating the present technique. We have calculated SFs and logarithmic derivatives for these data. Note that brightness is roughly representative of the density structure in the excited $\mathrm{H}_{2}$. In Fig. 4 the structure function of order 5 for the brightness and 

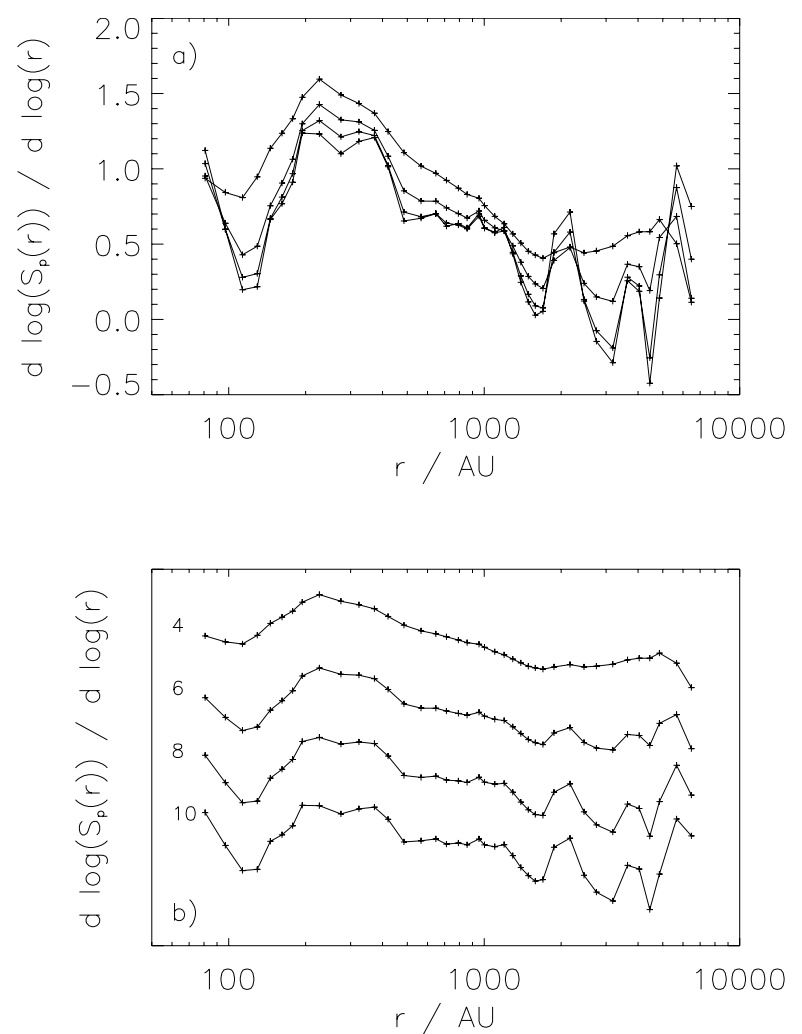

Fig. 5. a) Local logarithmic derivatives of structure functions of order 4 , $6,8,10$. b) As in a) but with the graphs displaced to get a better view of the evolution.

the corresponding derivatives are shown. Relative errors on values in the SFs are found using the law of propagation of errors for uncorrelated variables. Using the notation of Eq. (1), the variance is calculated as:

$$
\begin{aligned}
\sigma^{2}\left(S_{p}(r)\right)= & \left(p^{2} / N^{2}\right) \sum\left(\sigma^{2}\left(A\left(r^{\prime}\right)\right)+\sigma^{2}\left(A\left(r^{\prime}-\delta\right)\right)\right) \\
& \times\left|A\left(r^{\prime}\right)-A\left(r^{\prime}-\delta\right)\right|^{2(p-1)}
\end{aligned}
$$

where the summation is performed over all pairs of pixels in the map that satisfy $|\delta|=r, \mathrm{~N}$ is the total number of such pairs and $\sigma^{2}\left(A\left(r^{\prime}\right)\right)$ is the variance of $A\left(r^{\prime}\right)$. Due to the large number of pixel pairs in our data, $2 \times 10^{7}$ to $4 \times 10^{9}$, the variances are very small. Typical values of the relative errors, $\sigma\left(S_{p}(r)\right) / S_{p}(r)$, are $10^{-5}$ in the 2 nd order SF, $10^{-4}$ in the 5 th order SF and $10^{-3}$ in the 10 th order SF. Thus the errors are negligible in SFs of order up to 10 , as well as in the logarithmic derivatives of these functions.

Local minima in the derivatives as a function of $\mathrm{r}$ are present at $r \sim 110 \mathrm{AU}$ and $r \sim 1700 \mathrm{AU}$, providing clear evidence that the brightness structure, and by implication the density distribution, in OMC1 shows preferred scales. The positions of the minima indicate that the (largely) shocked regions in the field have two preferred sizes close to 110 and 1700 AU. Two less pronounced minima or points of inflection are present at $r \sim 550 \mathrm{AU}$ and $r \sim 2700 \mathrm{AU}$, which suggests that clumping at these scales is also present in the field, but to a lesser degree. As noted earlier these sizes may be representations of diameters in near-circular clumps or major and minor axes in elliptically shaped clumps.

In Fig. 5a the logarithmic derivatives of SFs of order 4, 6, 8, 10 are displayed for the same data. This illustrates the tendency for the derivatives of higher order SFs to congregate at the same value, a property mentioned in Sect. 2 . In Fig. 5 b the derivatives are displaced on the ordinate to give a clearer view of the
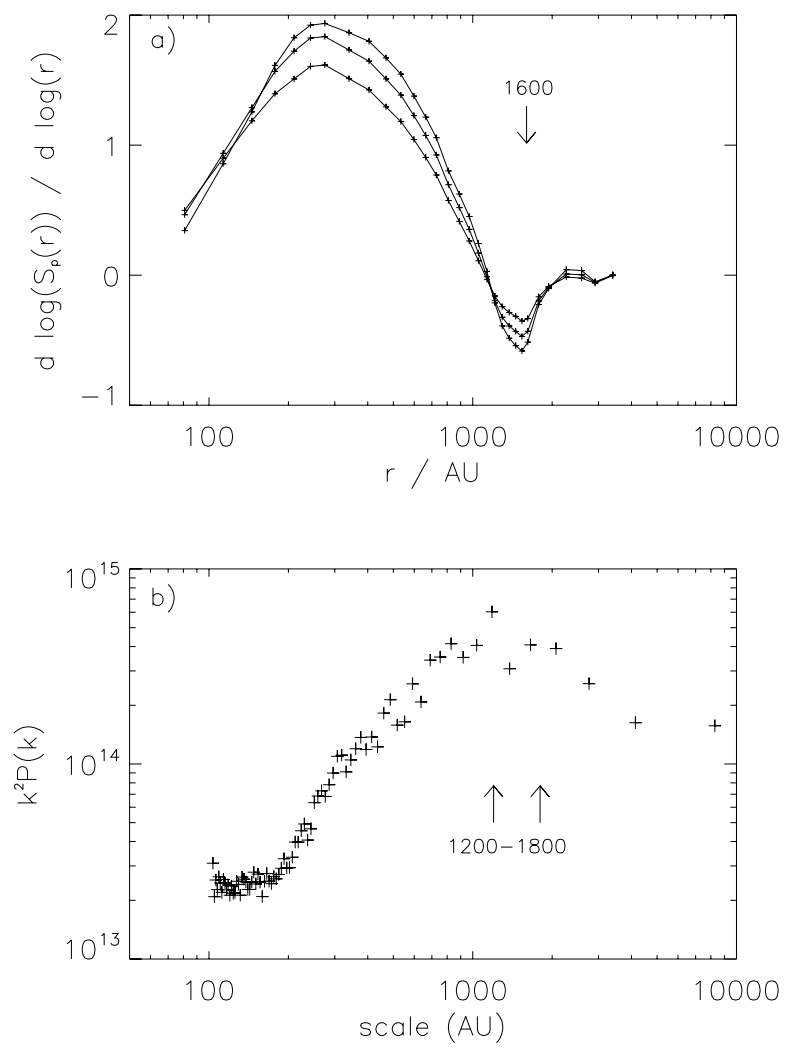

Fig. 6. a) Local logarithmic derivatives of structure functions of order 4, 5, 6 of a region South-West of BN in OMC1 calculated using CFHT/PUEO-KIR data. b) Fourier transform of the same set of data: the power spectrum multiplied by $k^{2}$ vs. the scale size.

evolution with regard to the order of the SF. As the order of the structure function increases, the derivatives show more detail. High order SFs accentuate the presence of weaker structure hidden in lower order SFs, resulting in more minima in the derivatives. Thus in the limit of very high order, so far as this can be achieved within the errors of observation, derivatives will essentially pick out the size of every clump in the region. This property is useful since the order of the structure function at which a structure becomes apparent is a measure of the prevalence of that structure. A structure that is clearly seen in the 2nd order SF is more dominant in the region than a structure which is first seen in the 6th order SF, say. Examples are the scale sizes at $r \sim 550 \mathrm{AU}$ and $2700 \mathrm{AU}$ which are barely visible in the derivatives of the 4th order SF, but are clearly seen in the derivatives of the 6 th order SF. These are less dominant than the scale sizes at $r \sim 110 \mathrm{AU}, 1700 \mathrm{AU}$, which are clearly apparent in the derivatives of the 4th order SF. In observational data, random errors in the calculated values will however increase when higher orders are computed, thus limiting the maximum value of $p$ that may be used. Thus uncertainties in observational data may generally be a limiting factor in this form of data analysis.

\subsection{Comparison with other methods using CFHT/PUEO-KIR and VLT/NACO data}

We first present a comparison of the method described here with the Fourier transform technique conducted on the CFHT/PUEO-KIR data of Kristensen et al. (2006). Figure 6a shows the derivatives of the SFs of order 4, 5, 6 of that region indicating a preferred scale of $1600 \mathrm{AU}$. We now compare this with the standard two-dimensional Fourier transform technique 


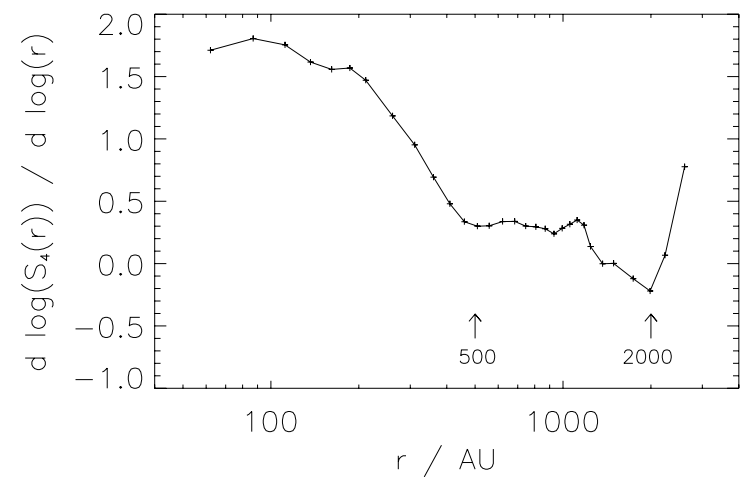

Fig. 7. Local logarithmic derivatives of the 4th order structure function of the ESE region analysed in Lacombe et al. (2004) from VLT/NACO data, comparable to the region analysed in Vannier et al. (2001).

commonly employed to identify scale size in images. This yields a power spectrum $P(k)$ which in the case of self-similar structure should be a power law in $k\left(k \propto r^{-1}\right)$. Deviations from a power law are nicely illustrated by showing $k^{2} P(k)$ vs. $k$, as described in Vannier et al. (2001). Figure 6b displays $k^{2} P(k)$ vs. scale size $\left(k^{-1}\right)$. Figure $6 \mathrm{~b}$ shows that there is a range of scales which are over-populated. This over-population peaks at $\sim 1200$ $1800 \mathrm{AU}$. The above results show good agreement between the scales obtained from the power spectrum and the SF derivatives, at any rate in this simple case in which only a single scale, or a single range of scales, is present in the region.

We now turn to a comparison with a more complicated example in which more than one scale is present. Preferred scales in $\mathrm{OMC} 1$ have previously been identified by Vannier et al. (2001) and Lacombe et al. (2004) using a variety of techniques. We now use the SF technique to obtain scale sizes in the VLT/NACO data. These may be compared with scale sizes reported in Lacombe et al. (2004) and Vannier et al. (2001). Using an area-perimeter analysis and a Fourier transform technique Vannier et al. (2001) found a preferred scale around $600 \mathrm{AU}$, but spanning a large and rather poorly determined range. Vannier et al. (2001) also used the brightness histogram technique of Blitz \& Williams (1997), finding a preferred scale of 900 AU. Lacombe et al. (2004), with the VLT/NACO data, used only the histogram technique finding a preferred size of $1100 \pm 130 \mathrm{AU}$.

The local derivatives of the 4 th order SF for the VLT/NACO data are shown in Fig. 7 where two scale sizes of $r \sim 500 \mathrm{AU}$ and $2000 \mathrm{AU}$ are evident. The lower of these scales is consistent with the findings in Vannier et al. (2001). As a further comparison, we created a two-dimensional Fourier transform to yield the power spectrum, $P(k)$ of the same field using the VLT/NACO rather than the $3.6 \mathrm{~m}$ La Silla data of Vannier et al. (2001). The power spectrum was - as it should be - very similar to that reported in Vannier et al. (2001) showing marked departure from a power law in $k$. Data in Fig. 7 show that the SF method is capable of much more precision in the identification of structure scale than a simple Fourier transform.

In summary, there is reasonably good correspondence between the standard technique of 2D Fourier transform and the SF method, so far as comparison can be made. Both show clear departure from self-similarity and a similar scale size, where this can be identified as in Figs. 6a and b. There seems to be some discrepancy between these two techniques and the histogram method, on the basis of work reported in Vannier et al. (2001) and Lacombe et al. (2004)
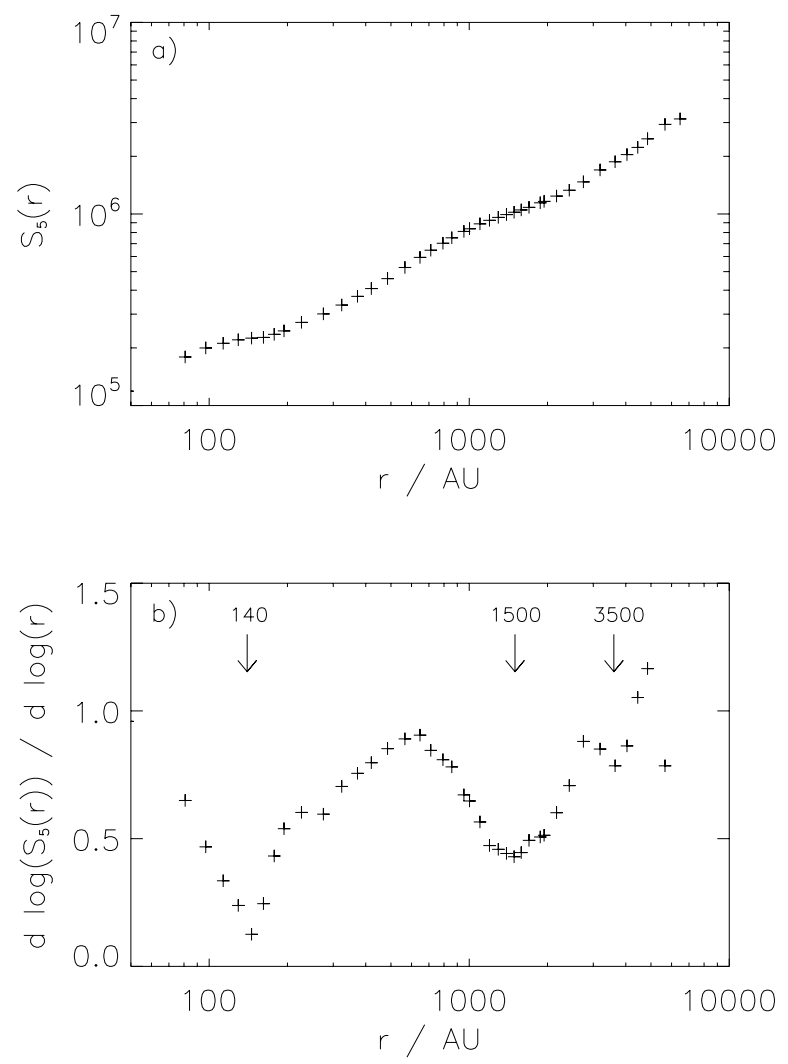

Fig. 8. a) The 5th order structure function of velocities in $\mathrm{OMC} 1$ from CFHT/GriF. b) Corresponding local logarithmic derivatives of the 5th order structure function.

\subsection{Use of the present method with $\mathrm{CFHT/GriF} \mathrm{H}_{2}$ velocity data}

We now return to the dataset covered by the CFHT/GriF observations, as described in Sect. 3. The 5th order structure function of the velocity data from GriF and the corresponding derivatives are shown in Fig. 8. Because the accuracy of the velocity in each pixel depends on the brightness in the pixel (see Sect. 3) each velocity difference in Eq. (1) has been weighted by the product of the brightness, $B$, in the two pixels in question, thus giving more weight to pixels with high brightness. The weighted SFs are thus

$S_{p}(r)=\left\langle B\left(r^{\prime}\right) B\left(r^{\prime}-\delta\right)\left|v\left(r^{\prime}\right)-v\left(r^{\prime}-\delta\right)\right|^{p}\right\rangle$.

Relative errors on the calculated values in the weighted SFs are typically $10^{-3}$ for fifth order $(p=5)$ and therefore negligible.

Figure 8 shows that local minima in the derivatives as a function of $\mathrm{r}$ are present at $r \sim 140 \mathrm{AU}, 1500 \mathrm{AU}$ and $3500 \mathrm{AU}$. This analysis therefore reveals that there is local order in the velocity field at these scales. It is interesting that these scales are respectively disk scales (140 AU) and outflow scales (>1000 AU), the latter being associated with (re-)injection of energy into the system, that is, a driving scale. These scales are approximately the same as those identified in the brightness data, which were found to be at 110 and $1700 \mathrm{AU}$, although there appears to be an additional larger scale at $3500 \mathrm{AU}$ in velocity. The weak structure in the brightness at $r \sim 550 \mathrm{AU}$ and $2700 \mathrm{AU}$ is however not seen in the velocity. An analysis of the velocity data has also been performed without brightness weighting with essentially the same result as in Fig. 8. 


\subsection{Systematic errors associated with estimates of structure scale}

There are systematic errors inherent in the present method. As already noted, derivatives of higher order SFs tend to pick out scales which are too small by as much as $10-20 \%$. It turns out that when we consider a distribution of clump sizes about some mean value, this causes an overestimation of the mean clump size as we discuss below. These two effects tend to cancel but to an extent which cannot be quantified.

The detection of a preferred scale size implies that there is a range of sizes around the derived value. In common with other methods, our method does not give any measure of the extent of such a range. Here we test the effect of introducing a distribution of clump sizes about some mean preferred scale using artificial images. In order to illustrate the behaviour of such systems, we assume that the sizes of clumps are Gaussian distributed about the mean. Using simulations similar to those in Sect. 2 we have created a number of maps with 200 randomly positioned clumps. Three mean sizes of 40, 60 and 80 pixels were used. We have found that when the half-width of the Gaussian distribution is smaller than $\sim 5 \%$ of the mean value, the SFs are indistinguishable from the original of only one clump size and the minimum of the SF derivatives is found at the mean value. Increasing the half-width beyond $\sim 5 \%$ causes the minimum to move towards larger scales. If the half-width of the distribution is $10 \%(20 \%)$ $25 \%$ of the mean value the minimum is found at $1.07(1.25) 1.50$ times the mean value. For example, if the data used in Fig. 6 consist of clumps distributed as a Gaussian with a halfwidth of $20 \%$ of the mean value, the detected value of $1600 \mathrm{AU}$ corresponds to a true mean value of the sizes of structures of $\sim 1300 \mathrm{AU}$. This analysis only conveys the sensitivity of the systematic error to the width of the distribution but does not allow an estimation of the width from observations. The distribution of clump sizes about some preferred scale is directly linked to the IMF and it would be useful to develop methods which were capable of making a reliable width estimate.

\section{Conclusion}

In conclusion the technique presented here, whilst suffering from some degree of uncertainty and imprecision to an extent common to all methods, has proved valuable for analysis of images in our own work. With the present and future advent of higher spatial resolution maps in the radio region of the spectrum, probing the star-forming scale, for example obtained with the Plateau de Bure interferometer, the Submillimeter Array, CARMA and ALMA, this tool may prove useful to an increasing community of observers.

Acknowledgements. M.G. and D.F. would like to acknowledge the support of the Instrument Center for Danish Astrophysics (IDA), funded by the Danish Natural Science Research Council and the Aarhus Centre for Atomic Physics (ACAP), funded by the Danish Basic Research Foundation.

\section{References}

Bally, J., O’Dell, C. R., \& McCaughrean, M. J. 2000, AJ, 119, 2919 Bensch, F., Stutzki, J., \& Ossenkopf, V. 2001, A\&A, 366, 636 Biferale, L., Boffetta, G., Celani, A., et al. 2004, Phys. Rev. Lett., 93, 064502 Blitz, L., \& Williams, J. P. 1997, ApJ, 488, L145

Boldyrev, S., Nordlund, Å., \& Padoan, P. 2002a, ApJ, 573, 678 Boldyrev, S., Nordlund, A.., \& Padoan, P. 2002b, Phys. Rev. Lett., 89, 031102 Chappell, D., \& Scalo, J. 2001, ApJ, 551, 712

Clénet, Y., Le Coarer, E., Joncas, G., et al. 2002, PASP, 114, 563

Elmegreen, B. G., \& Scalo, J. 2004, ARA\&A, 42, 211

Falgarone, E., \& Phillips, T. G. 1990, ApJ, 359, 344

Farge, M. 1992, Ann. Rev. Fluid Mechanics, 24, 395

Frisch, U. 1995, Turbulence. The legacy of A.N. Kolmogorov (Cambridge: Cambridge University Press)

Gustafsson, M., Kristensen, L. E., Clénet, Y., et al. 2003, A\&A, 411, 437

Gustafsson, M., Field, D., Lemaire, J. L., \& Pijpers, F. P. 2006, A\&A, 445, 601

Kolmogorov, A. N. 1941, Dokl. Akad. Nauk, 30, 301

Kristensen, L. E., Gustafsson, M., Field, D., et al. 2003, A\&A, 512, 727

Kristensen, L. E., Ravkilde, T., Field, D., Lemaire, J. L., \& Pineau des Forêts 2006, A\&A, submitted

Kritsuk, A. G., \& Norman, M. L. 2004, ApJ, 601, L55

Lacombe, F., Gendron, E., Rouan, D., et al. 2004, A\&A, 417, L5

Larson, R. B. 1981, MNRAS, 194, 809

Mac Low, M., \& Klessen, R. S. 2004, Reviews of Modern Physics, 76, 125

Nissen, H., Gustafsson, M., Lemaire, J., et al. 2005, A\&A, to appear [arXiv: astro-ph/0511226]

Ossenkopf, V., \& Mac Low, M.-M. 2002, A\&A, 390, 307

Padoan, P., \& Nordlund, Å. 2002, ApJ, 576, 870

Rouan, D., Field, D., Lemaire, J.-L., et al. 1997, MNRAS, 284, 395

Stutzki, J., \& Guesten, R. 1990, ApJ, 356, 513

Stutzki, J., Bensch, F., Heithausen, A., Ossenkopf, V., \& Zielinsky, M. 1998, A\&A, 336, 697

Vannier, L., Lemaire, J. L., Field, D., et al. 2001, A\&A, 366, 651 